\title{
Retain or sacrifice the posterior cruciate ligament in total knee arthroplasty? A histopathological study of the cruciate ligament in osteoarthritic and rheumatoid disease
}

\author{
R G H H Nelissen, P C W Hogendoorn
}

\begin{abstract}
Background-The decision whether to retain or resect the posterior cruciate ligament in total knee arthroplasty is at present determined clinically by preoperative radiological variables focusing upon the amount of joint destruction, and subsequent soft tissue contractures. However, these variables give only indirect information on the histological integrity and proprioceptive properties of the posterior cruciate ligament.

Methods-Twenty posterior cruciate ligaments, obtained during total knee arthroplasty, were evaluated histologically to study the relation between the degree of preoperative radiological joint destruction, structural integrity of the posterior cruciate ligament and the neurological integrity of the targeted tissue. Eleven patients had osteoarthritis and nine patients rheumatoid arthritis. Haematoxylin and eosin, Alcian blue (mucoid degeneration), elastica von Gieson, Gomori (elastic fibres and collagen), and immunohistochemical staining for neural structures were used. Results-In all but one of the posterior cruciate ligaments, morphologically intact neural tissue was present in the peritendineum of the ligaments. Structural integrity of the collagen framework was present in only seven posterior cruciate ligaments. These cases all had grade three or four radiological joint destruction. In 13 of the specimens a certain degree of mucoid degeneration of collagen was present. All patients with grade five radiological knee joint destruction displayed mucoid degeneration and irregularity of the posterior cruciate ligament fibres.

Conclusion-Because of the extensive architectural and probably functional damage of the posterior cruciate ligament in patients who have grade five radiological knee joint destruction, retention of the posterior cruciate ligament in knee prosthesis should not be advocated. (F Clin Pathol 2001;54:381-384)
\end{abstract}

Keywords: cruciate ligaments; knee prostheses; histology; innervation

The posterior cruciate ligament has received much attention in total knee arthroplasty; whether to resect or retain it remains controversial. Arguments are often based on certain theoretical advantages, and the importance of femoral roll back in the presence of the posterior cruciate ligament. ${ }^{1-3}$ However, Mahoney and colleagues ${ }^{4}$ showed that, after total knee arthroplasty, the physical strain on the ligament was different to that seen in preoperative normal knees, and femoral roll back was reduced. In addition to the biomechanical function of the posterior cruciate ligament, one has to consider its potential proprioceptive action. Innervation of the normal posterior cruciate ligament is derived from the popliteal plexus, which receives branches from the posterior articular nerve (a branch of the posterior tibial nerve) and from the obturator nerve. ${ }^{56}$ Some authors reported that proprioception was decreased after posterior cruciate ligament resection, ${ }^{137}$ whereas others found no further worsening of position sense after cruciate sacrificed total knee arthroplasty. ${ }^{8}$ Furthermore, some authors ${ }^{9}$ even showed that position sense could improve after cruciate sacrificed total knee arthroplasty.

Excision of the cruciate ligaments in a normal knee joint will alter the biomechanics of the knee joint. ${ }^{10}$ However, it is ambiguous to state that the biomechanics of the (severely) degenerative knee joint will be altered in time, with subsequent adaptations (that is, degeneration) of the knee ligament structure. With this knowledge, two problems remain: how to recognise the "normal" posterior cruciate ligament and whether it is possible to create the optimal tensioned cruciate ligament. The first issue, to identify which posterior cruciates ligament are suitable for retention, will be investigated here. The decision on whether to resect or retain the posterior cruciate ligament is currently made preoperatively or based on clinical factors intraoperatively. Because an in depth analysis of the biomechanical and proprioceptive properties of the posterior cruciate ligament at that time is impossible, we compared radiological knee joint destruction with the properties of the posterior cruciate ligament to identify objective preoperative parameters influencing decision making related to preservation of the posterior cruciate ligament.

\section{Materials and methods}

PATIENTS

Twenty posterior cruciate ligaments were removed during surgery for a cruciate sacrificing, posterior stabilised knee prosthesis (InsallBurstein II; Zimmer, Warsaw, Indiana, USA). This study comprises a consecutive series of knee prostheses. Because at that time only a
Accepted for publication 20 September 2000 
Table 1 Descriptive statistics of 20 posterior cruciate ligaments in degenerative knees

\begin{tabular}{llllllll}
\hline Patient & $\begin{array}{l}\text { Age } \\
\text { (years) }\end{array}$ & $\begin{array}{l}\text { Sex } \\
(M / F)\end{array}$ & Diagnosis & $\begin{array}{l}\text { Preoperative } \\
\text { destruction }\end{array}$ & Collagen fibre & $\begin{array}{l}\text { Bone } \\
\text { fragments }\end{array}$ & Neuro-filaments \\
\hline 1 & 69 & F & OA & 4 & Focal mucoid/irregular & - & + \\
2 & 85 & F & OA & 5 & Extensive mucoid/irregular & - & + \\
3 & 60 & F & OA & 5 & Focal mucoid/irregular & - & + \\
4 & 73 & F & OA & 5 & Extensive mucoid/irregular & + & - \\
5 & 63 & M & OA & 5 & Focal mucoid/irregular & - & + \\
6 & 77 & F & OA & $3 / 4$ & None/parallel & - & + \\
7 & 84 & F & OA & 4 & None/local irregular & - & + \\
8 & 75 & F & OA & 4 & Slight mucoid/local irregular & - & + \\
9 & 79 & F & OA & 5 & Extensive mucoid/irregular & + & + \\
10 & 79 & M & OA & 5 & Focal mucoid/irregular & - & + \\
11 & 82 & F & OA & 4 & None/local irregular & - & + \\
12 & 49 & F & RA & 4 & None/slight irregular & - & + \\
13 & 69 & F & RA & $3 / 4$ & None/parallel & - & + \\
14 & 82 & F & RA & 4 & None/slight irregular & + & + \\
15 & 36 & F & RA & 4 & None/parallel $/$ & + \\
16 & 45 & F & RA & 5 & Focal mucoid/slight irregular & + & + \\
17 & 85 & F & RA & 5 & Slight mucoid/parallel & + & + \\
18 & 66 & F & RA & 5 & Slight mucoid/parallel & - & + \\
19 & 55 & F & RA & 4 & Slight mucoid/parallel & - & + \\
20 & 48 & F & RA & 5 & Focal mucoid/irregular & - & + \\
\hline
\end{tabular}

Diagnosis: OA, osteoarthritis; RA, rheumatoid arthitis.

Preoperative destruction: classification according to Ahlback (OA) or Larsen (RA). In patients six and 13 a difference was present between the medial and lateral compartments.

Collagen fibres: mucoid degeneration (slight, focal, or extensive, see text) or normal aspect. Irregular or parallel collagen fibre orientation.

M, male; F, female.

posterior cruciate ligament sacrificing knee prothesis was used, no patient selection was made. Eleven patients suffered from osteoarthritis, the other nine patients suffered from rheumatoid arthritis. The degree of radiological osteoarthritis was classified preoperatively as grade one to five (grade five being a deformed knee without joint space) on an anteroposterior weight bearing radiograph of the knee, as detailed previously. ${ }^{11}{ }^{12}$ All ligaments were sharply excised from their tibial and femoral attachments, marking the femoral side of the posterior cruciate ligament.

Four normal posterior and anterior cruciate ligaments were obtained at necropsy from a 38 year old male patient, who had no history of degenerative joint disease and who had macroscopically normal cartilage of the knee joint. These ligaments were used as reference sections for the ligaments of the degenerative joints.

HISTOLOGY

All specimens were preserved immediately in $4 \%$ buffered formaldehyde solution and processed and embedded in paraffin wax according to routine laboratory practice before being cut. The specimens were cut in parallel $3 \mu \mathrm{m}$ thick sections.

The slides were stained with haematoxylin and eosin, Alcian blue (to detect mucoid degeneration of collagen fibres), Gomori stain, or elastica von Gieson (for visualisation of collagen fibre orientation). ${ }^{13}$ The structural properties and integrity of the posterior cruciate ligament were analysed with these specific elastic, collagen fibre, and mucoid (degeneration) staining techniques. Neural structures in the posterior cruciate ligament were assessed using immunohistochemical staining techniques. ${ }^{14}$ These techniques use antibodies directed against neurofilaments and S-100 protein (Dako, Glostrup, Denmark; 1/2000 dilution). Histological examination was implemented by conventional light microscopy.

\section{Results}

\section{PATIENTS}

The patients ranged from 36 to 85 years old (mean, 68; SD, 14.9). The study group consisted predominantly of women. Preoperatively, at least a radiological grade three to five destruction of the knee joint was present in all cases (table 1).

\section{HISTOLOGICAL APPEARANCE}

The reference sections from four postmortem normal ligaments showed the presence of undulated neurofilaments, parallel fibre orientation, and no mucoid degeneration. A synovial sleeve composed of a uniform single cell layer with subsynovial fat tissue was present around these ligaments.

In all but one posterior cruciate ligament specimen, neural structures were identified (figs 1 and 2). The axons observed had an undulated appearance, with a parallel orientation with respect to the collagen fibres (fig 1). The single posterior cruciate ligament without detectable neural structures was present in an osteoarthritic knee of a 73 year old female patient with grade five radiological osteoarthritis.

Three of the posterior cruciate ligaments had a normal appearance and, in another four only

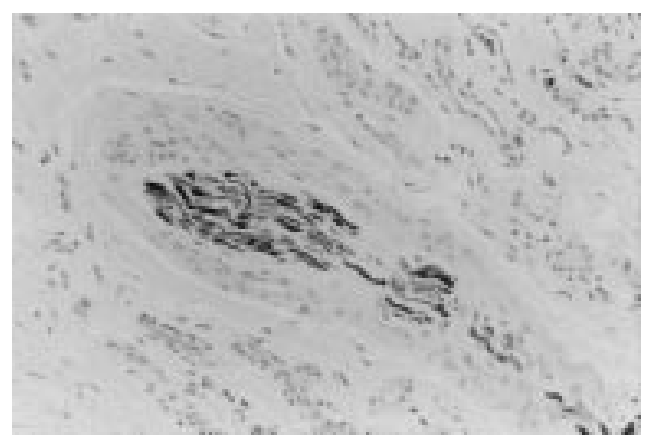

Figure 1 Light micrograph of an immunohistochemical slide, showing an axon with undulated neurofilaments $(S-100$; original magnification, $\times 40)$. 


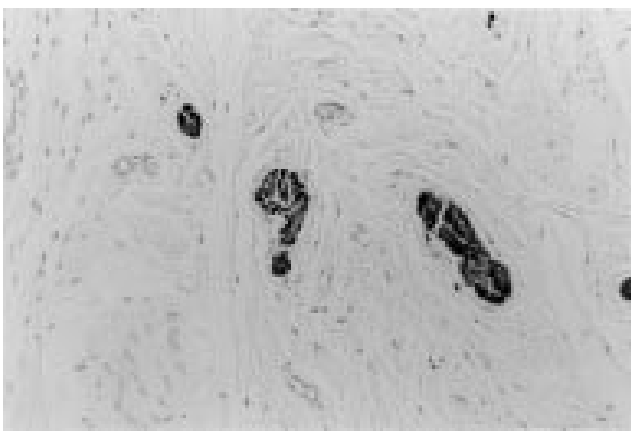

Figure 2 Light micrograph of an immunohistochemical slide, showing a cross section of axon in perisynovial tissue $(S-100$; original magnification, $\times 40)$.

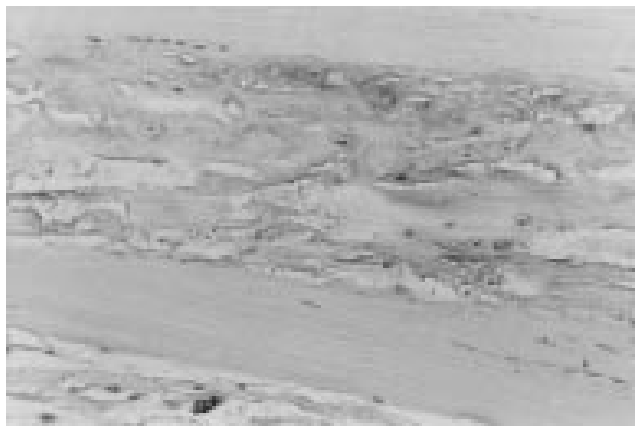

Figure 3 Light micrograph showing focal mucoid degeneration and parallel orientation of collagen fibres (Alcian blue stain; original magnification, $\times 40$ ).

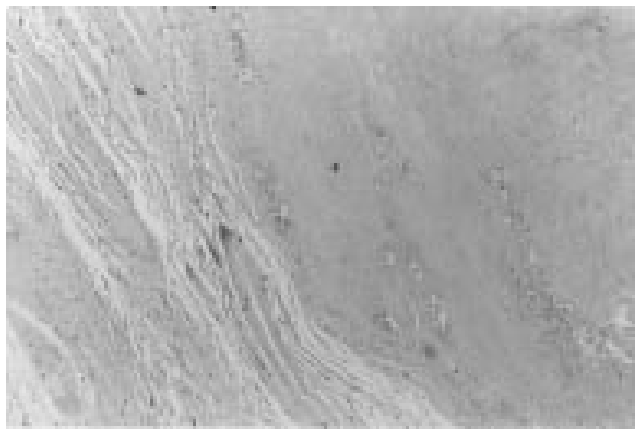

Figure 4 Light micrograph showing extensive mucoid degeneration and irregularity of collagen fibres (Alcian blue stain; original magnification, $\times 20$ ).

a slight irregular orientation of the collagen fibres was noted (table 1). In the seven cases with normal or slight irregularity of collagen deposition, grade three to four radiological kneejoint destruction was present.

Rheumatoid arthritic ligaments showed a thickened hyperplastic synovial cell layer, vascular proliferation, hemosiderin deposition, lymphoid aggregates, and histiocytes dispersed between the collagen fibres.

In both osteoarthritic and rheumatoid arthritic cruciate ligaments small bone fragments were found in six posterior cruciate ligaments.

In 13 of the specimens, a certain degree of mucomyxoid degeneration was found (figs 3 and 4). Several stages of mucoid degeneration were present, namely: (1) early stage, with slight degeneration starting between the collagen bundles and collagen bundles still having a normal parallel arrangement; (2) moderate foci of mucoid degeneration with irregular structure of the collagen fibres; and (3) extensive mucomyxoid degeneration with total loss of fibre structure and bundle integrity. All posterior cruciate ligaments in grade five radiological knee destruction had focal to extensive mucoid degeneration with irregular fibre orientation (figs 3 and 4). In most specimens (14 of the posterior cruciate ligaments), the fibre structure of the cruciate ligaments was irregular. Only six cruciate ligaments had a normal parallel fibre orientation.

\section{Discussion}

Functional integrity of the posterior cruciate ligament is essential for cruciate retaining knee prostheses. Furthermore, these prostheses could benefit from the proprioceptive properties of the intact posterior cruciate ligament. However, posterior cruciate ligaments with a normal appearance were present in only three knee joints with grade three or four radiological knee joint destruction. Most of the posterior cruciate ligaments had extensive mucoid degeneration and irregular collagen fibre orientation. These extensive changes in the structural properties of the posterior cruciate ligament were present in radiological knee joint destruction grade five as well. However, intact neurological properties were present in all but one posterior cruciate ligament (grade five radiological destruction).

Morphological studies concerning the posterior cruciate ligament of the knee have been performed with non-specific neural staining techniques, ${ }^{15-18}$ thus overestimating or underestimating neural tissue present in the ligament. We used histochemical/immunohistochemical staining techniques to analysis joint innervation. Previously used conventional gold and silver chloride staining techniques are not specific to neural tissue, but stain vessels as well. ${ }^{19} 20$ The immunohistochemical techniques used in our study provide specific information about quantitative, well functioning innervation because they are directed against neurone specific neurofilament subunits that stabilise a functioning axonal cytoskeleton. The presence of neural tissue in the posterior cruciate ligament means that under certain conditions (stretch of proprioceptors), the mechanical state of the ligament is monitored by the central nervous system. The balance between hamstrings (sciatic nerve) and quadriceps (femoral nerve) is regulated by this additional proprioceptive information from the knee joint. Contradicting reports that show no effect, ${ }^{8}$ a decreased, ${ }^{1}$ or an improved position sense after posterior cruciate ligament retained knee prosthesis might be explained by the differences in ligamentous integrity found in our study (that is, bone fragment incorporation, extent of mucoid degeneration). These differences create different viscoelastic properties and probable differences in triggering of the neurofilaments. In all but one posterior cruciate ligament specimen neurofilaments were present. The undulated structure of the axon in some of the arthritic and in the normal posterior cruciate ligament specimens (that is, parallel to the collagen fibrils) is probably related to the stretch sensitivity of neurofilaments. 
The presence of neural structures in most posterior cruciate ligaments of degenerative knee joints was in contrast to the findings of other authors. ${ }^{16}{ }^{17}$ This discrepancy might result from the different staining techniques used: non-specific gold chloride ${ }^{16-18}$ versus neural specific immunohistochemistry techniques.

The histological degenerative changes of the posterior cruciate ligament (mucoid degeneration, incorporation of bone fragments) in most of the specimens probably result from synovitis of the arthritic knee ${ }^{21}$ and mechanical microtraumata, with subsequent avulsion of bone fragments from the degenerative joint surface. The small bone fragments found in the posterior cruciate ligament might be forced into the ligaments mechanically (during knee movement), rather than arising by metaplastic bone formation from the constitutional cells of the ligament, which have a different microscopic morphology.

Histological differences between osteoarthritic and rheumatoid arthritic ligaments in this series were present mainly in the synovium. The rheumatoid arthritic ligaments were characterised by hyperplasia and/or hypertrophy of the synovial layer. In osteoarthritic posterior cruciate ligaments more extensive mucoid degeneration was present.

Disrupted and weakened collagen fibres in cruciate ligaments have also been found by others, but no description of the extent of the degenerative ligamentous change was given. ${ }^{17}{ }^{21}$ In our study, posterior cruciate ligament mucoid degeneration was present in three distinct phases (slight, moderate, and extensive), which have been related to the extent of radiological knee destruction.

We therefore conclude that excision or retention of the posterior cruciate ligament during total knee arthroplasty should be based not only on clinical evaluation of the knee, but also on the degree of radiological knee destruction, which parallels the functional state of the posterior cruciate ligament as assessed histologically. Knees with a grade three to four radiological destruction had a virtually normal structural integrity of the cruciate ligament and would thus ideally fit a posterior cruciate ligament retaining total knee arthroplasty. A posterior cruciate ligament retaining knee prosthesis should not be used in the severely damaged knee joint (radiological stage five) because advanced structural changes compromise the functional capacity of the posterior cruciate ligament.

1 Andriacchi TP, Galante JO, Fermier RW. The influence of total knee-replacement design on walking and stairclimbing. F Bone foint Surg Am 1982;64:1328-35.

2 Andriacchi TP, Stanwyck TS, Galante JO. Knee biomechanics and total knee arthroplasty. F Arthroplasty 1986;1: $211-19$

3 Soudry M, Walker PS, Reilly DT, et al. Effects of total knee replacement design on femoro-tibial contact conditions. $\mathcal{F}$ Arthroplasty 1986;1:35-45.

4 Mahoney OM, Noble PC, Rhoads DD, et al. Posterior cruciate function following total knee arthroplasty. F Arthroplasty 1994;9:569-77.

5 Gardner E. The innervation of the knee joint. Anat Rec 1948;101:109-30.

6 Kennedy JC, Alexander IJ, Hayes KC. Nerve supply of the human knee and its functional importance. Am $\mathcal{F}$ Sports Med 1982;10:329-35.

7 Simmons S, Lephart S, Rubash $\mathrm{H}$, et al. Proprioception following total knee arthroplasty with and without the posterior cruciate ligament. F Arthroplasty 1996;11:763-7.

8 Skinner HB, Barrack RL, Cook SD, et al. Joint position sense in total knee arthroplasty. F Orthop Res 1984;1:276-

9 Barrett DS, Cobb AG, Bentley G. Joint proprioception in normal osteoarthritic and replaced knees. F Bone foint Surg Br 1991;73:53-6.

10 Girgis FG, Marshall JL, Al Monajem ARS. The cruciate ligaments of the knee joint. Clin Orthop 1975;106:216-31.

11 Ahlbäck S. Osteoarthrosis of the knee. A radiographic investigation. Acta Radiol Diagn 1968;277(suppl):7-72.

12 Larsen A, Dale K, Eck M. Radiographic evaluation of rheumatoid arthritis and related conditions by standard reference films. Acta Radiol 1977;18:481-91.

13 Gigante A, Specchia N, Greco F. Age-related distribution of elastic fibers in the rabbit knee. Clin Orthop 1994;308:3342.

14 Enzinger FM, Weiss SW. Immunohistochemistry of soft tissue lesions In: Soft tissue tumors. Washington DC: Mosby, 1995:139-65.

15 Franchi A, Zaccherotti, Aglietti P. Neural system of the human posterior cruciate ligament in osteoarthritis. 7 Arthroplasty 1995;10:679-82.

16 Hagena FW, Hofmann GO, Mittlmeier T, et al. The cruciate ligaments in knee replacement. Int Orthop 1989;13:13-16.

17 Schultz RA, Miller DC, Kerr CS, et al. Mechanoreceptors in human cruciate ligaments. F Bone foint Surg Am 1984;66: 1072-6.

18 Schutte MJ, Dabezies EJ, Zimny ML, et al. Neural anatomy of the human anterior cruciate ligament. $\mathcal{F}$ Bone foint Surg Am 1987;69:243-7.

19 DeAvila GA, O'Connor BL, Visco DM, et al. The mechanoreceptor innervation of the human fibular collateral ligament. F Anat 1989;162:1-7.

20 Gómez-Barrena E, Martnez-Moreno E, Munuera L. Segmental sensory innervation of the anterior cruciate ligament and the patellar tendon of the cat's knee. Acta Orthop Scand 1996;67:545-52.

21 Goldberg VM, Burstein A, Dawson M. The effect of an experimental immune synovitis on the failure mode and strength of the rabbit anterior cruciate ligament. F Bone foint Surg Am 1982;64:900-5. 